combination of IgM-RF $>40$ or anti-CCP $>50$, with a sensitivity of $55.4 \%$ and a specificity of $96.7 \%$.

\begin{tabular}{lll} 
Abstract THU0141 Table 1 & & \\
\hline & Specificity (\%) & Sensitivity (\%) \\
\hline IgM-RF > 30 & 93.4 & 50.4 \\
IgA-RF > 10 & 94.2 & 52.7 \\
Anti-CCP > 50 & 97.5 & 42.6 \\
IgM-RF > 40 & 96.7 & 46.5 \\
IgM-RF > 30 and IgA-RF > 10 & 96.7 & 46.5 \\
IgM-RF > 40 or anti-CCP > 50 & 96.7 & 55.4 \\
\hline
\end{tabular}

Conclusion The criterion IgM-RF $>40$ or anti-CCP $>50$ is able to predict the onset of RA with a high specificity and acceptable sensitivity. Anti-CCP testing provides additional information on which to base treatment decisions in early arthritis.

\section{THU0142 RELATION BETWEEN NTX AND CARF IN RHEUMATOID ARTHRITIS}

M Oh, H Kikuchi, M Saitoh, H Nisisaka, T Matusita, S Sohen. Orthopaedic Surgery, Sakai Hospital Kinki University School of Medicine, Sakai, Japan

\subsection{6/annrheumdis-2001.1044}

Background It is well known that cytokines cause abnormal immunity affecting bone resorption in rheumatoid arthritis (RA). NTx (typeI collagen cross-linked N-telopeptides) is bone resorption marker, CARF (Anti-agalactosyl IgG antibodies) is a new rheumatoid factor which is able to detect all RF isotype, and allows early diagnosis of the onset of RA.

Objectives To find out the bone resorption in RA, we investigated the relation between NTx and CARF, inflammatory parameters CRP and ESR, Lansbury's joint count which is the score for the affected joint.

Methods NTx and CARF were measured in 31 RA patients (male: 1 case, female:30 cases). And CRP, ESR, Lansbury's joint count was measured at the time, and examined about that relationship.

Results Significant positive correlations were observed between NTx and CARF ( $\mathrm{r} 2=0.211 \mathrm{p}=0.0093)$, and between NTx and joint count $(\mathrm{r} 2=0.154 \mathrm{p}=0.0321)$, however, no significant correlations were observed between NTx and CRP or ESR. Conclusion These results suggest that NTx has a potentiality as the joint destruction marker, reflecting not only general inflammation but also local inflammation such as arthritis.

\section{THU0143 ASSOCIATIONS OF WALKING ABILITY TO ISOKINETIC KNEE EXTENSOR AND FLEXOR STRENGTH IN WOMEN WITH RHEUMATOID ARTHRITIS (RA)}

${ }^{1}$ OR Madsen, ${ }^{2} \mathrm{C}$ Egsmose. ${ }^{1}$ Department of Rheumatology, Herlev and Amager University Hospitals, Herlev; ${ }^{2}$ Department of Rheumatology, Bispebjerg and Gentofte University Hospitals, Copenhagen, Denmark

\subsection{6/annrheumdis-2001.1045}

\section{Background}

Objectives

Methods Seventy-five women with RA according to the 1987 ACR criteria were examined. Mean age was $62 \pm 13$ years, median disease duration 11 (1-51) years. Sixty-three were or had been on steroids [median cumulative prednisolone dose 2.5 (0.05-66) g]. Sixty-seven age-, weight- and height-matched healthy women served as controls. Maximal voluntary knee extensor and flexor strength $(\mathrm{Nm})$ was assessed at $30 \mathrm{o} / \mathrm{s}$ by an isokinetic dynamometer. Walking ability was expressed as walking and stair climbing speed $(\mathrm{m} / \mathrm{s})$. Markers of disease activity included number of swollen and tender joints, pain as recorded by the patients on a visual analogue scale (VAS), and disability as scored by the Stanford Health Assessment Questionnaire (HAQ). Statistics: non-parametric.

Results Muscle strength, walking speed and stair climbing speed were on average reduced by $30 \%, 28 \%$ and $54 \%$ ( $\mathrm{p}<0.0001$ ), respectively, compared to the controls. Significant correlations were found between knee extensor strength and walking speed $(\mathrm{r}=0.79, \mathrm{p}<0.0001)$ and stair climbing speed $(\mathrm{r}=0.69, \mathrm{p}<$ 0.0001). Similar correlations were found for flexor strength. The correlations remained significant (Rpartial ranging from 0.64 to $0.69, \mathrm{p}<0.0001$ ) in multiple regression analyses adjusting for age, height, weight, disease duration, number of swollen and tender joints, VAS- and HAQ-score.

Conclusion In conclusion, leg muscle strength is considerably reduced and is an important determinator of walking ability in RA.

\section{THU0144 ASSOCIATION OF ANTIPHOSPHOLIPID ANTIBODIES WITH RETINAL VASCULAR DISEASE IN REUMATOID ARTHRITIS}

B Seriolo, V Ferretti, D Fasciolo, A Sulli, M Cutolo. Division of Rheumatology. Department of Internal Medicine and Medical Specialities, University of Genova, Genova, Italy

\subsection{6/annrheumdis-2001.1046}

Background Rheumatoid arthritis (RA) may affect any organ of the body and display a broad spectrum of clinical and immunological manifestations. Ocular involvement has been described less commonly and includes involvement by mucocutaneous disease, secondary Sjogren's syndrome, neuro-ophthalmic lesions, and retinal vascular disease.

Objectives The aim of our study was to analyse the possible association between the presence of antiphospholipid antibodies (aPL) and retinal lesions detected by means of prospective ophthalmologic examination in a series of RA patients.

Methods Sixty-eight female RA patients attending our Extraarticular Involvement RA Clinic were studied. All patients fulfilling the 1987 American Rheumatism Association Criteria for adult RA. Ophthalmologic examination included assessment of best corrected visual acuity, tonometry, slit-lamp bimicroscopy, and fundus examination. Serologic studies included determination of anticardiolipin antibodies (aCL) (ELISA) and Lupus Anticoagulant (LA) (Coagulation Test).

Results Retinal vascular disease was detected in 7 (10\%) of 68 RA patients. The retinal lesions consisted of retinal vascular occlusion in two patients (one arterial and one venous thrombosis), cotton-wool sports in one, optic disc oedema in two cases, retinal haemorrhages in one, and ischaemic optic neuropathy in one case. aPL were detected in $5(71 \%)$ of these 7 patients: four had aCL and one had LA. When compared with patients without retinal vascular disease, patients with retinopathy had a higher prevalence of aPL (71\% vs $6 \%) ; \mathrm{p}=0.0001)$. 